\title{
DECADENCIA Y RESURGIMIENTO DEL GANADO EQUINO EN LA REGIÓN DE MURCIA
}

\author{
Cayetano Espejo Marín \\ Carmen García Frago \\ Andrés Cuenca Sánchez
}

\section{RESUMEN}

La utilización del ganando equino ha ido perdiendo importancia en la actividad agrícola a medida que ésta se ha ido mecanizando. Así el ganado mular y asnal prácticamente han desaparecido mientras que el caballar inicia una recuperación sostenida desde 1975, vinculada a actividades deportivas y de ocio que generan nuevos tipos de explotaciones y de asociaciones ganaderas, a la vez que alcanza una importancia creciente en el movimiento comercial pecuario.

Palabras clave: ganado equino, explotación ganadera, comercialización, asociacionismo ganadero.

\section{RÉSUMÉ}

L'utilisation du bétail équin a progressivement perdu de son importance au sein de l'activité agricole au fur et à mesure que celle-ci s'est mécanisée. C'est ainsi que le cheptel mulassier et asinien ont pratiquement disparu tandis que le cheptel chevalin connaît un renoveau constant depuis 1975, renoveau lié à des activités sportives et de plaisance donnant naissance à de noveaux types d'exploitations et d'associations d'éleveurs, tout en atteignant une importance croissante dans le cadre du mouvement commercial concernant le bétail.

Mots clés: Bétail équin, exploitation d'élevage, commercialisation, associacionisme d'éleveurs.

El ganado equino tuvo mucha importancia en la ganadería murciana hasta mediados del siglo actual. Fundamental en otras épocas por su utilización para las diferentes tareas agrícolas, ha ido perdiendo progresivamente su importancia debido al paulatino proceso de mecanización de las mismas, estando hoy en día en proceso de extinción, sobre todo el asnal y mular, ya que el caballar, en los últimos años ha incrementado su censo. 


\section{Evolución de la cabaña equina}

A mediados del siglo XVIII el Catastro de Ensenada cifra en el año 1756 un total de 3.929 cabezas de caballar, 13.301 de mular y 27.585 de asnal.

La incidencia del ganado equino en el espacio murciano era importante, y destacaba de un modo especial en la actual comarca del Noroeste $^{1}$, donde los términos de Bullas, Caravaca, Cehegín y Moratalla, en su conjunto acumulan el 12,5 por 100 del total caballar, 22,5 por 100 del mular y 16 por 100 del asnal. El hecho de que nos encontremos en un espacio con una agricultura extensiva justifica la fuerte presencia del ganado mular. Sin embargo, no todo el mular es utilizado en labores de la tierra, tal y como se desprende de las propias declaraciones que realizan los dueños de los equinos. Así, mientras en Bullas sólo el 40 por 100 de las cabezas de ganado mular se ocupan en labor, en Moratalla son el 46,7 por 100, y en Caravaca tres de cada cinco mulos se destinas a esta tarea.

La estructura de la propiedad pecuaria de estos equinos presentaba importantes contrastes, frente a un reducido número de propietarios que poseen un número de cabezas superior al centenar, la mayoría disponen de sólo una. En Caravaca 4 dueños concentran el 56,2 por 100 del ganado mular, con un total de 875 cabezas, y en Cehegín, sólo 3, con 578 animales acumulan el 74 por 100 del ganado mular de este término. Frente a estos grandes propietarios, en todos los términos, a excepción de Caravaca, la categoría predominante es la de una sola cabeza, aunque el mayor número se incluyen en el grupo de las de dos mulos, utilizados como yunta.

En el Campo de Lorca los aparceros igualmente solían tener un par de bestias, generalmente burras o mulas, para laboreo de la tierra, trilla y tiro de carros, sin perjuicio de que los más modestos con reducidas parcelas a su cargo, dispusiesen de un sólo jumento y viniesen obligados a redoblar su esfuerzo físico, mientras los más acomodados, que llevaban las mayores explotaciones de regadío, contaban con varias yuntas, a veces bueyes, utilizados asimismo para el enganche de carretas ${ }^{2}$.

Con la comparación de las cifras registradas en el Catastro de Ensenada y en los Censos Ganaderos de mediados del siglo XIX, se observa un incremento muy importante en las tres clases de equino:

\begin{tabular}{|cccc|}
\hline & Caballar & Mular & Asnal \\
\hline 1756 & 3.929 & 13.301 & 27.585 \\
1859 & 6.083 & 34.033 & 48.465 \\
1865 & 11.028 & 51.562 & 81.454 \\
\hline
\end{tabular}

El desarrollo de la ganadería siguió la línea ascendente iniciada en la segunda mitad del siglo XVIII, es decir no hubo ruptura una vez superada la hecatombe originada por la Guerra de la Independencia. El crecimiento ganadero tuvo lugar en un periodo de ampliación de las superficies cultivas ${ }^{3}$.

1 ANDRÉS SARASA, J.L. y ESPEJO MARÍN, C. (1987-89): «La cabaña ganadera en el noroeste murciano a mediados del siglo XVIII», Estudios Románicos. Homenaje al Profesor Luis Rubio, vol. 6, pp. 1.5211.534 .

2 GIL OLCINA, A. (1990): «Lorca según las Respuestas Generales del Catastro de Ensenada», en Lorca. 1755. Respuestas Generales del Catastro de Ensenada, Madrid, Tabapress, p. 34.

3 PÉREZ PICAZO, M.T. y LEMEUNIER, G. (1984): El proceso de modernización de la Región Murciana (siglos XVI-XIX), Murcia, Editora Regional de Murcia, pp. 353-355. 
En general, el ganado equino estaba asociado a las actividades agrícolas y al acarreo y transporte de mercancías. La importancia de las transformaciones agrícolas y del crecimiento del producto agrario desde mediados del siglo XIX supuso el uso creciente del equino hasta comienzos del siglo XX. La tónica general fue el crecimiento global de los efectivos orientados al trabajo hasta 1914. Igualmente se producen modificaciones importantes como la sustitución del asno por el mulo o el caballo, según la capacidad adquisitiva de los campesinos y sus necesidades laborales ${ }^{4}$.

En el último tercio del siglo XIX continúa el aumento del ganado de tiro, lo que sigue siendo exponente de que la agricultura aún está alcanzando cada día mayor auge al tiempo que ganaba superficie a costa de algunas zonas de monte. Durante el siglo XIX el núcleo más importante de la riqueza pecuaria en algunas comarcas, como el Altiplano, lo formaron los ganados de tiro, sobre todo el mular y el asnal ${ }^{5}$.

Las líneas básicas de especialización de la ganadería murciana se orientan a cubrir las necesidades del trabajo agrícola y del consumo alimenticio. En el caso de las primeras se produce una gran expansión del mular y caballar en la segunda mitad del siglo XIX y las dos primeras décadas del siglo XX, aumentando su peso relativo en el conjunto de la cabaña española ${ }^{6}$.

En el diario «El Liberal» se describe, a través de su director, la incidencia del ganado asnal en las ferias que se celebran en la ciudad de Murcia a comienzos del siglo XX: «La vuelta que di hace unos días por la feria de ganados, me hizo observar como predominaba en él sobre todos los animales útiles para la agricultura, la mansa, la clásica, la bíblica pollina. Efectivamente está justificado el gran número de esos animales que traen los feriantes, porque la demanda que se hace es de ellos. En la Huerta de Murcia son pocos los colonos que no tienen una burra» ${ }^{7}$.

En el año 1920 se establece en Fuente Álamo de Murcia la Feria de Ganados del 1 al 5 de Junio, anunciada como «Gran Feria anual de ganado lanar, mular, caballar, asnal y de cerda. Conocida es la situación inmejorable de este pueblo para esta clase de ferias. Cuenta con frondosos arbolados y aguas abundantes. El sitio donde ha de instalarse la feria está dotado de varios abrevaderos. Para el ganado que concurra se han reservado cuarenta fanegas de pastorado» ${ }^{8}$.

Con el paso de los años la citada feria de ganados va adquiriendo cada vez más notoriedad y a mediados de siglo se publica en el semanario «Murcia Sindical» la crónica siguiente: «De la importancia de esta feria el visitante puede hacerse una idea con sólo ver o saber que desde el puente de la carretera a Murcia, que cruza por encima de la rambla y sirve de emplazamiento a la referida Feria y que la domina, sólo se ve a ambos lados de ella y del puente, una casi interminable fila de ganados caballar, mular, asnal y vacuno de todas clases; carros típicos valencianos, tratantes y vendedores vestidos con sus no menos típicas blusas»?.

La evolución de las tres clases de ganado equino desde 1960 hasta el momento actual queda reflejada en la figura 1.

4 MARTÍNEZ CARRIÓN, J.M. (1991): La ganadería en la economía murciana contemporánea. 18601936, Murcia, Consejería de Agricultura, Ganadería y Pesca, pp. 49-50. 310 .

5 MORALES GIL, A. (1972): El Altiplano de Jumilla-Yecla, Murcia, Universidad de Murcia, pp. 309-

6 MARTÍNEZ CARRIÓN, J.M., Op. cit., 1991, p. 59.

7 MARTÍNEZ TORNEL, J.: El Liberal, 8-9-1907.

8 ORTEGA MERINO, R. (1991): Crónica de Fuente Álamo, Ayuntamiento de Fuente Álamo, p. 274.

9 MURCIA SINDICAL, 5 de Junio de 1949. 


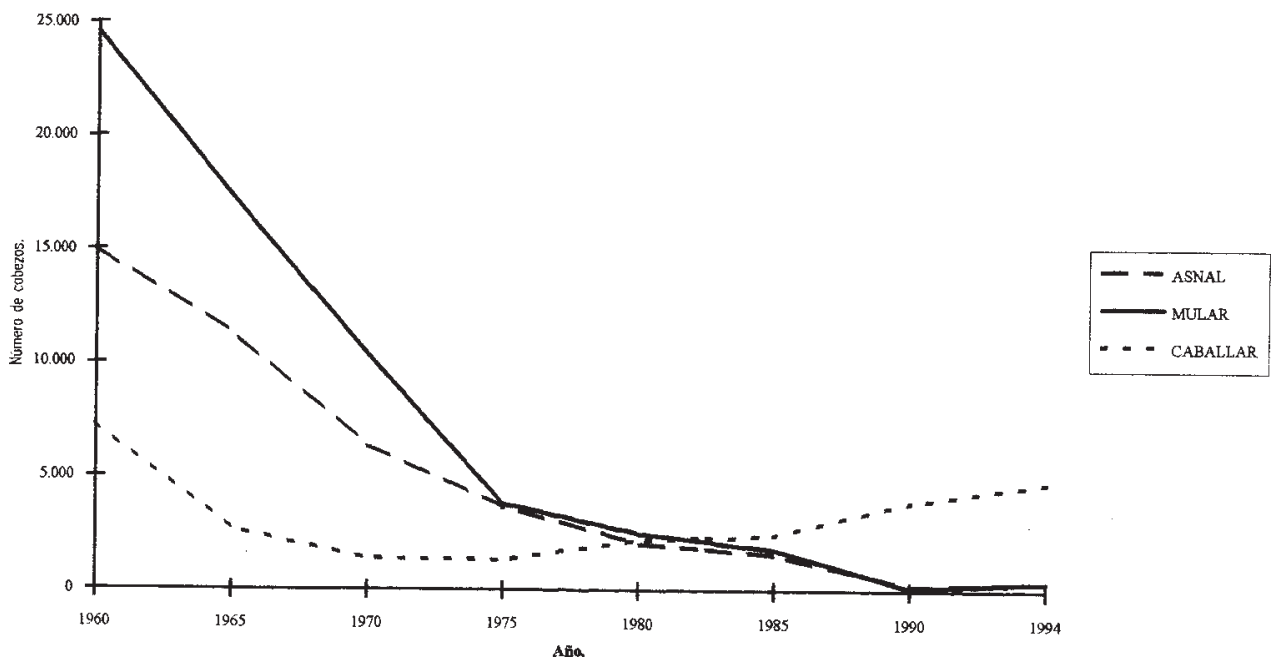

Figura 1. Evolución de la Cabaña Equina en la Región de Murcia. 1960-1994.

De su observación se desprende como mular y asnal prácticamente han desaparecido, mientras que el caballar recupera su censo a partir de 1975 y sigue creciendo hasta hoy.

Durante la segunda mitad del siglo XX, el total del ganado de labor registra unas pérdidas que equivalen a dividir por ocho las existencias de 1950; pero este divisor se alcanza por el incremento del caballar, ganado que hoy es utilizado para actividades bien distintas a la agricultura. En cambio, mular y asnal, animales específicamente de tiro, llevan camino de quedar muy pronto reducidos a meras reliquias, fenómeno que ha ya experimentado la raza autóctona de la vaca murciana ${ }^{10}$.

En España, en los años cuarenta y cincuenta la riqueza de los campesinos se cuantificaba más por el número de yuntas disponibles que por la tierra que con ellas se trabajaba. Y, no faltaba la palabra expresiva de quienes sólo disponían de una yunta: eran los pegulajeros o yunteros, los que dentro de la escala social campesina, componían un tramo solamente superior al de los gañanes y temporeros. El ganado estaba muy supeditado a la agricultura: la especie equina y, en buena medida, también la bovina se explotaban sobre todo para el acarreo o el transporte de los frutos y el arrastre del arado, del trillo o de la carreta. La primera igualmente, para el volteo de la noria y, con su montura, como único medio de comunicación en el medio rural ${ }^{11}$.

Esta situación descrita para el ámbito nacional, es la misma que se da en nuestra Región durante estos años, tal y como se desprende de los estudios realizados sobre el Campo de Lorca y el Altiplano de Jumilla-Yecla.

En el Campo de Lorca el censo ganadero mostraba un marcado predominio, sobre el caballo, de la mula y el asno, animales rústicos y poco exigentes en pienso. Los dos últimos dedicados desde siempre a las faenas agrícolas, mientras el ganado caballar se reservaba

10 ANDRÉS SARASA, J.L. y ESPEJO MARÍN, C. (1989): «La mecanización de la agricultura en la Región de Murcia», Revista de Estudios Agrosociales, núm. 147, pp. 156-157.

11 CABO ALONS0, A. (1993): «La cabaña española en el último medio siglo», en Medio siglo de cambios agrarios en España, Alicante, Instituto de Cultura «Juan Gil-Albert», p. 116. 
preferentemente al tiro de carruajes y para, mediante el cruce con garañón, obtener muletas castellanas. La mula era el animal de trabajo más caracterizado, sobre todo en los secanos lorquinos. La general predilección por las yuntas de mulas se justificaba por que a la fuerza y resistencia se unían la ligereza de marcha ${ }^{12}$.

En la comarca del Altiplano, hasta los años sesenta, las especies que predominaban dentro del censo ganadero eran la mular y el asnal. La primera estaba muy cotizada por lo bien que soportaba los trabajos del campo y daba mayor rendimiento por la ligereza de su marcha y la resistencia a la fatiga. El ganado asnal se utilizaba sobre todo para el transporte a lomo, siendo típicas las reatas de burros que transportaban la leña a los hornos o cualquier otro producto que hubiera que llevarse por senderos de montañas ${ }^{13}$.

Hoy en día nadie pone en duda que la caída de los censos de ganado equino se deben a la creciente mecanización de las actividades agrícolas. Desde mediados del presente siglo en todas las comarcas de la Región de Murcia, tal y como sucede en la del Altiplano, se inició un profundo cambio en el utillaje agrícola, pues se pasó de la más elemental utilización manual de algunos instrumentos, y del movido con tracción animal, a la introducción de aperos de tracción motorizada. El proceso se inició en las dos primeras décadas - cincuenta y sesenta - de forma lenta y pausada, ya que la carencia de capitalización suficiente por parte de la mayoría de los agricultores y su tendencia a una economía agrarias basada en explotaciones familiares, impidieron su acceso a la motorización ${ }^{14}$.

\section{Distribución espacial de las explotaciones}

La distribución espacial del ganado equino a escala municipal en la actualidad se conoce en función del modo de explotación o aprovechamiento que se hace del mismo; por ello tenemos por un lado las explotaciones equinas, que se definen como el conjunto de instalaciones empleadas para la cría del ganado con fines de producción y/o reproducción. En segundo lugar contamos con los núcleos zoológicos-establecimientos para la práctica de la equitación, y que son los que albergan équidos con fines recreativos, deportivos o turísticos, incluyen picaderos, cuadras de alquiler y otros centros para la práctica ecuestre.

El Registro Regional de Explotaciones Equinas contabiliza un total de 71 explotaciones, que se distribuyen sobre el espacio regional tal y como se observa en la figura 2 . Se trata de explotaciones dedicadas a la producción de carne, reproducción, y/o actividades de compraventa. Lorca y Murcia, ambos municipios suman cerca de la mitad del total regional de explotaciones equinas. Cartagena y Torre Pacheco son los otros términos donde se dan con un número un poco significativo, ya que en el resto su número no supera las dos explotaciones, y como se puede observar son bastantes los casos en donde el ganado equino ha desaparecido por completo.

El Registro de Núcleos Zoológicos relaciona un total de 42, repartidos en un tercio del total de los municipios de la Región (Figura 3). Murcia y Cartagena, son los que presentan mayor número, 11 y 7 respectivamente. En los demás la cifra no supera los 3 establecimientos para la práctica de la equitación.

12 GIL OLCINA, A. (1971): El Campo de Lorca. Estudio de Geografía Agraria, Valencia, Departamento de Geografía de la Universidad de Valencia y C.S.I.C., p. 190.

13 MORALES GIL, A., Op. cit., 1972, p. 318. 


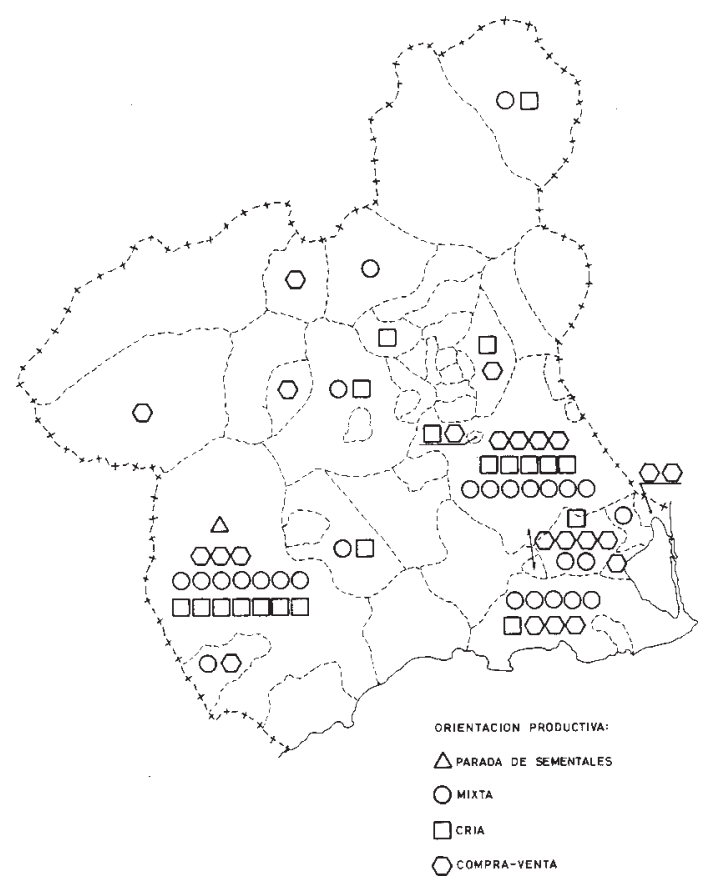

FIGURA 2. Distribución de las explotaciones equinas. 1994.

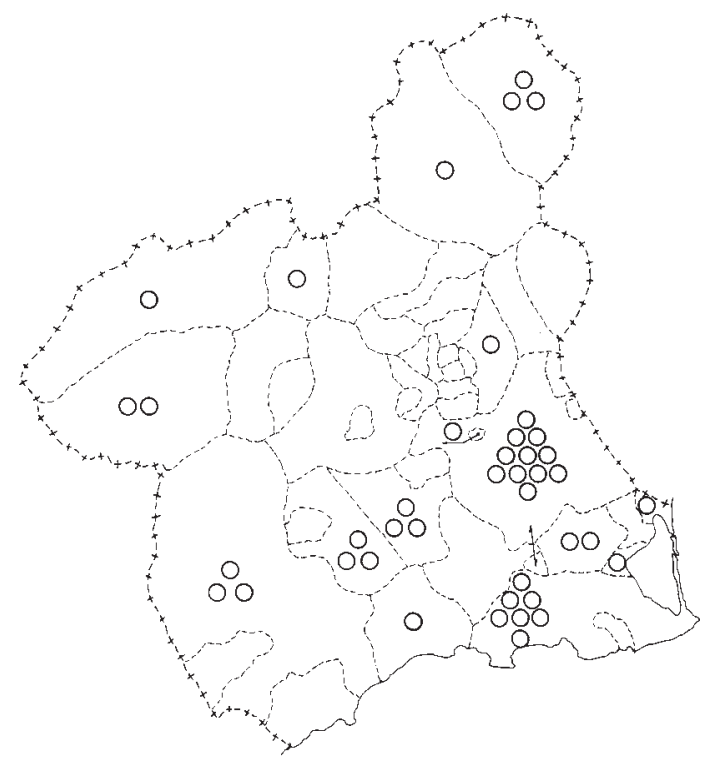

FIGURA 3. Distribución de los núcleos zoológicos con actividad equina. 1994. 


\section{Movimiento comercial pecuario}

Los intercambios internacionales de caballos, reproductores de raza pura y con caballos destinados a otros usos, mueven progresivamente mayores cifras tanto en la vertiente importadora como en la exportadora en los países con cierto nivel ecuestre.

En España, el ganado caballar tiene aún por consolidar su presencia en el exterior por lo que sus resultados, aunque han mejorado en estos últimos años, están muy alejados de los óptimos, dadas las magníficas ganaderías con las que se cuenta y que permiten augurar un futuro esperanzador ${ }^{15}$.

Uno de los datos que contribuyen a explicar el resurgimiento de la actividad equina en la Región de Murcia es el que hace referencia a los intercambios comerciales.

En diciembre de 1993 España es declarada libre de «Peste Equina Africana», lo que permite la reactivación de los movimientos de animales con destinos y procedencias de los más variados lugares.

El ganado equino en la Región de Murcia es el que presenta un menor volumen de cabezas comercializadas. No obstante, a partir de 1994 se producen dos hechos a tener en

Cuadro I

MOVIMIENTO COMERCIAL PECUARIO. 1994

\begin{tabular}{|c|c|c|c|c|}
\hline & \multicolumn{2}{|c|}{ DESTINO } & \multicolumn{2}{|c|}{ PROCEDENCIA } \\
\hline & Cabezas & $\%$ & Cabezas & $\%$ \\
\hline GALICIA & & & 1 & 0,02 \\
\hline CANTABRIA & 1 & 0,02 & & \\
\hline ARAGÓN & 10 & 0,17 & 11 & 0,18 \\
\hline CATALUÑA & 22 & 0,37 & 12 & 0,20 \\
\hline BALEARES & 2 & 0,03 & 3 & 0,05 \\
\hline CASTILLA-LEÓN & 5 & 0,08 & 9 & 0,15 \\
\hline CASTILLA-LA MANCHA & 201 & 3,40 & 17 & 0,28 \\
\hline COMUNIDAD VALENCIANA & 1.518 & 25,68 & 1.142 & 18,73 \\
\hline EXTREMADURA & & & 156 & 2,56 \\
\hline ANDALUCÍA & 318 & 5,38 & 1.170 & 19,19 \\
\hline REGIÓN DE MURCIA & 3.210 & 54,31 & 3.210 & 52,65 \\
\hline ALEMANIA & 27 & & 9 & \\
\hline BÉLGICA & 30 & & 7 & \\
\hline FRANCIA & 537 & & 37 & \\
\hline TOTAL & 594 & 10,05 & 53 & 0,87 \\
\hline TOTAL & 5.911 & 100 & 6.097 & 100 \\
\hline
\end{tabular}

Fuente: Movimiento Comercial Pecuario.

14 MORALES GIL, A. y BOX AMORÓS, M. (1993): «Cambios agrarios en las comarcas de transición del sureste peninsular a La Mancha», en Medio siglo de cambios agrarios en España, p. 568.

15 LARREA EREÑO, S. (1996): «El mercado exterior del caballo», El Campo, núm. 135, p. 251. 
cuenta. El primero es el fuerte incremento de las cabezas vendidas respecto a años anteriores, en su práctica totalidad de ganado caballar. En el año 1989 se venden un total de 1.399 cabezas, la casi totalidad de ellas en las explotaciones ${ }^{16}$. En cambio, un quinquenio más tarde el número de equinos vendidos es de 5.911, y de éstos una parte muy importante se comercializan a través de ferias ganaderas dedicadas al caballo con carácter monográfico.

La mitad de las cabezas transaccionadas proceden y tienen como destino el espacio regional. El resto preferentemente tienen su origen o van a parar a las comunidades autónomas limítrofes, y sobre todo a las provincias de Alicante, Almería, Albacete y Granada.

En el año 1994 la Región de Murcia presenta un saldo comercial positivo con los países de la Unión Europea. Destaca el hecho de que tengan este destino el 10 por 100 del total de cabezas vendidas, siendo Francia el país que recibe el mayor número (Cuadro I).

\section{Asociacionismo y fomento del ganado caballar}

Como consecuencia del desarrollo la que está adquiriendo el ganado caballar en la Región de Murcia surgen con fuerza asociaciones que tienen como objetivo el control y desarrollo de esta especie. Dos son las más importantes.

La Asociación de Criadores de caballos de Razas Puras de la Región de Murcia se constituye en marzo de 1988, y surge como consecuencia del constante aumento de ganaderías de equinos de puras razas en la Región de Murcia y ante la necesidad de aunar esfuerzos, con el fin de consolidar y mejorar en lo posible la cabaña equina. En total fueron 20 ganaderos los fundadores de esta Asociación, bajo la coordinación del entonces coronel de Caballería, Delegado de Cría Caballar, D. Juan Díaz de Figueroa y Soriano, de quien partió la idea de crear la misma. En número de cabezas de ganado en aquella fecha eran de 18 machos y 67 hembras de pura raza española y 2 hembras de Pura raza árabe.

La idea matriz de la asociación es el fomento de la colaboración entre los asociados y contar con una representación autorizada ante los Organismos Oficiales.

En el año 1995 cuenta la Asociación con 76 socios, que son propietarios de un total de 210 hembras y 181 machos de pura raza española, y 13 hembras y 4 machos de pura raza árabe.

Con el fin de mantener informados a los asociados, de lo que acontece en el mundo del caballo, se crea en 1989 la revista «Nuestros Caballos».

En la actualidad la asociación está orientando sus esfuerzos a la consolidación de un Concurso Morfológico que se pretende sea representativo de la Cría Caballar en la Región de Murcia y que sirva de estímulo a los ganaderos en su labor de selección. Del mismo modo se pretende completar la actividad de este Concurso, con una forma moderna y atractiva de Muestra Ganadera, que permita activar y reforzar los canales comerciales existentes y abrir nuevos mercados.

La Asociación para el Fomento del Caballo, con sede en Lorca, se constituye en septiembre de 1995 y la forman un grupo de 20 ganaderos con el fin de fomentar el mundo del caballo y no sólo el de pura raza, también el cruzado, del que reivindican sus cualidades. Entre los objetivos de esta Asociación está el de dar impulso a las dos ferias que se celebran en Lorca especialmente dedicadas al mundo del caballo. En ambas se muestran caballos tanto en exposición como en venta.

16 ESPEJO MARÍN, C. (1996): Comercialización y producción ganadera en la Región de Murcia, Murcia, Consejería de Medio Ambiente, Agricultura y Agua, p. 78. 
Uno de los proyectos en los que está trabajando la Asociación para el Fomento del Caballo es en la creación de un Centro Tecnológico del Caballo. El fomento del turismo rural a caballo es otro de los objetivos de la Asociación, ya que el desarrollo de esta actividad permite rentabilizar los caballos.

Con el aumento en los últimos años de los caballos de pura raza española, así como de su extraordinaria difusión geográfica, en nuestro país han sido muchos los eventos ecuestres que se han desarrollado. Como consecuencia de ello se ha producido un importante acercamiento del caballo al gran público, lo que hace pensar que nos encontramos ante un sector que cada día cuenta con una consolidación empresarial y un potencial cada vez mayor $^{17}$.

La Región de Murcia no ha quedado al margen del desarrollo de estas eventos con ganado caballar. Durante los años 1994 y 1995 se realizan un total de 33 y 30 actos respectivamente. Se establecen tres tipos de actividades.

En el primer grupo se incluyen las concentraciones, exhibiciones, pasacalles y romerías. En ambos años suponen la mayoría de los eventos, 22 en 1994 y 15 en 1995. En segundo lugar se incluyen los concursos hípicos, espectáculos ecuestres y saltos de obstáculos, su número se repite en ambos años y asciende a media docena. Por último están las ferias dedicadas con carácter monográfico al ganado caballar, y que son encuentros donde se realizan preferentemente transacciones comerciales. En 1994 se organizan 4 ferias y su número se eleva a 7 en 1995, hecho que contribuye a explicar el auge de la actividad ganadera caballar.

Los municipios en los que se organizan todos estos eventos son muy pocos, una decena en cada uno de los años, y de ellos 7 participan en 1994 y 1995: Murcia, Torre Pacheco, Cartagena, Lorca, Caravaca, Moratalla y San Pedro del Pinatar, mientras que Fuente Álamo y Jumilla que organizan en 1994, en 1995 lo hacen Mazarrón y San Javier.

Otro hecho a tener cuenta es que los municipios en los que se dan las actividades, ambos años, además de presentar varios tipos de actos, se repite el número de los mismos según la clasificación establecida, por lo que podemos hablar del comienzo de la consolidación de todos estos eventos.

Las fechas con mayor volumen de acontecimientos tienen en los meses de marzo y abril su máximo, aunque en el resto de los meses, salvo en el período invernal, también se dan con cierta incidencia las actividades relacionadas con el ganado caballar.

\section{Conclusiones}

De todo lo dicho podemos concluir que el ganado equino en general ha ido sufriendo un proceso de retroceso en su importancia dentro de la ganadería regional paralelo a la progresiva mecanización de la agricultura, perdiendo así su principal función de animal de trabajo.

De las tres especies que componen el ganado equino: caballar, mular y asnal, sólo el caballar ha aumentado su censo, en relación con su utilización para actividades de ocio.

Las otras dos especies: mular, dedicado en exclusiva al trabajo, y asnal utilizado como medio de transporte han experimentado una evolución totalmente negativa hasta su casi desaparición. Sólo se mantienen en algunas pequeñas explotaciones con carácter residual, cuyos propietarios los mantienen por apego a la tradición.

El ganado caballar durante los últimos años experimenta un gran auge, y para su consolidación y desarrollo surge un nuevo asociacionismo ganadero.

17 FERNÁNDEZ DE LA VEGA DE SANTOS, V. (1996): «Ferias, concursos morfológicos y eventos ecuestres», El Campo, núm. 135, pp. 81-89. 
